\title{
ASSESSING PEDOTRANSFER FUNCTIONS TO ESTIMATE THE SOIL WATER RETENTION
}

\author{
VALIDAÇÃO DE FUNÇÕES DE PEDOTRANSFERÊNCIA PARA ESTIMATIVA DA \\ RETENÇÃO DE ÁGUA NO SOLO
}

\section{Bruno Teixeira RIBEIRO ${ }^{1}$; Adriana Monteiro da $\operatorname{COSTA}^{2}$; Bruno Montoani SILVA ${ }^{1}$; Fernando Oliveira FRANCO ${ }^{3}$; Camila Silva BORGES ${ }^{1}$}

1. Departamento de Ciência do Solo, Universidade Federal de Lavras - UFLA, Lavras, MG, Brasil. brunoribeiro@dcs.ufla.br; 2. Instituto de Geociências, Departamento de Geografia, Universidade Federal de Minas Gerais - UFMG, Belo Horizonte, MG, Brasil; 3. Empresa de Pesquisa Agropecuária de Minas Gerais, Unidade Oeste, Uberaba, MG, Brasil.

\begin{abstract}
Considering the importance of soil water retention for agricultural and environmental purposes, the objective of this study was to assess three pedotransfer functions (PTFs) used to estimate the soil moisture at field capacity (FC) based on soil attributes easily determined. A collection of 17 soils from the Cerrado and Pantanal biomes, including surface and subsurface horizons, was used. PTF-1 considers clay, organic matter, coarse sand, and microporosity; PTF-2 clay, total sand, and organic matter; and PTF-3 only microporosity. The estimated FC values were correlated to soil moisture values measured at different soil water potentials $(0,6,10,33,100,300$, and $1500 \mathrm{kPa})$ to verify which potential corresponded to estimated FC. The data were subjected to regression analysis and Mann-Whitney rank-sum test to compare predicted and measured values and to principal component analysis (PCA). The analysis of the full dataset indicated that there was a strong correlation ( $\mathrm{R} 0.84-0.91 ; \mathrm{R}^{2} 0.71-0.82$; RMSE 0.07-0.09) between estimated FC and soil water retention measured at potentials of $10 \mathrm{kPa}$ and $33 \mathrm{kPa}$. FC estimated by PTF-3 correlated better with water holding capacity at $6 \mathrm{kPa}$. When the PTFs were reapplied to homogeneous soil groups (identified by PCA analysis), the correlation between predicted and measured FC was decreased.
\end{abstract}

KEYWORDS: Soil-water retention. Principal component analysis. Soil moisture.

\section{INTRODUCTION}

Water retention in soils has a crucial agronomic and environmental importance. Several phenomena depend on soil water retention, including plant growth and nutrient absorption, leaching of nutrients and pollutants, irrigation and drainage, hydrological recharge and modeling, and biochemical processes and microbial activity, among others. Water retention is determined and modeled in laboratory conditions; however, its measurement is time-consuming and costly, especially in tropical climates, where the soil properties vary widely and data are scarce (HARTEMINK, 2002; COSTA et al., 2013; BOTULA et al., 2014).

The upper limit of soil water content available to plants is known as field capacity (FC). FC has been defined as the soil water content remaining after free drainage is negligible (TOLK, 2003) and can be determined in situ or measured in the laboratory (VEIMEHYER; HENDRICKSON, 1949). FC is quantified in the laboratory using undisturbed soil samples saturated with distilled water and equilibrated at a soil water potential of 6 , 10 , or $33 \mathrm{kPa}$ (REICHARDT, 1988; RUIZ et al., 2003; KLEIN et al., 2006). The remaining water content corresponds to FC. Nonetheless, a consensus about FC estimation has not been reached (REICHARDT, 1988; SOUZA; REICHARDT, 1996; SILVA et al., 2014). In Brazil, a potential of $10 \mathrm{kPa}$ has been largely used for sandy and clayey soils with granular microstructure. The primary soil attributes related to FC are texture, structure, and organic matter (AULER et al., 2017). A coarse soil texture and low organic matter content result in low FC (RAWS et al., 2003; TOMASELLA et al., 2000; COSTA et al., 2013). Moreover, the size, distribution, and connectivity of pores as affected by soil structure and management strongly affect FC.

Pedotransfer functions (PTFs) have helped overcome the difficulties in estimating FC by using soil data that are easily measured and strongly associated with FC (PIDGEON, 1972; BOUMA, 1989; LOOY et al., 2017). PTFs are also available for tropical soils, including those in Brazil (MACEDO, 1991; TOMASELLA et al., 2000; REICHERT et al. 2009; COSTA et al., 2013; SANTOS et al., 2013; SOARES et al., 2014). However, much effort is still needed to accurately predict soil hydraulic properties in the tropics (MINASNY; HARTEMINK, 2011; BOTULA et al., 2014). Accurate use of PTFs depends on their thorough validation under different conditions and in this sense, Macedo et al. (2002) recommended to use their equations for sand, loamy sand, and sandy 
loam soil samples. We choose PTFs because the soil attributes used in these functions are easily determined, and physical data on Pantanal soils are limited. In this context, this study aimed to estimate FC in distinct soils samples from the Pantanal and Cerrado biomes, using three PTFs equations.

\section{MATERIAL AND METHODS}

This study was carried out using several surface and subsurface horizons of 14 soils from the Cerrado and Pantanal biomes in Brazil, totaling 69 soil samples. The soils were classified and studied during the 2012 Brazilian Meeting of Soil Classification and Correlation, Mato Grosso do Sul state, Brazil (RBCC, 2012) (Table 1). The soil attributes used in this study are shown in Table 2. The original data are available in the Field Handbook for the Soils of Pantanal and Cerrado, Mato Grosso state, Brazil (RBCC, 2012).

The volumetric soil moisture at $\mathrm{FC}$ was estimated using the three PTFs proposed by Macedo (1991) and Macedo et al. (2002):

$$
\begin{aligned}
& \mathrm{FC}=-0.01 \mathrm{C}-0.37 \mathrm{~S}+1.36 \mathrm{OM}-0.02 \mathrm{CS}+0.19 \mathrm{MC}+42.20 \\
& \mathrm{FC}=0.05 \mathrm{C}-0.45 \mathrm{~S}+1.80 \mathrm{OM}+49.39 \\
& \mathrm{FC}=0.80 \mathrm{MC}+2.32
\end{aligned}
$$

where $\mathrm{FC}$ is field capacity (\% by volume), $\mathrm{C}$ is clay content (\%), OM is organic matter content (\%), CS is coarse sand (\%), and MC is microporosity (\%).

The estimated FC values obtained by each PTF $(1,2$, and 3) were correlated with real soil water contents determined at different potentials $(0$, $6,10,33,100,300$, and $1500 \mathrm{kPa})$. The soil water retention curve was built according to Embrapa (2011) using a potential table and Richards's chamber.

The Shapiro-Wilk test was used to check the normality of data. The measured and estimated data were compared using Student's $t$-test (for normal data) or Mann-Whitney rank-sum test (for nonnormal data). In this study, Student's $t$-test (or the
Mann-Whitney rank-sum test) compared two data groups (estimated and measured data). These tests assess whether the mean estimated FC values are statistically equal to the mean measured $\mathrm{FC}$ values (FABIAN; OTTONI FILHO, 2000).

Principal component analysis (PCA) was performed using the software Statistica. PCA was used to identify soil groups according to the analyzed soil attributes (Table 2) and to calculate the predicted soil moisture content using the three PTFs with the remaining soil moisture at different potentials.

\begin{tabular}{|c|c|c|c|}
\hline $\begin{array}{c}\text { Soil } \\
\mathrm{n}^{\circ}\end{array}$ & Soil & Horizon & Layer $(\mathrm{cm})$ \\
\hline 1 & Orthic Humiluvic Spodosol & Eko & $0-6$ \\
\hline 2 & Orthic Humiluvic Spodosol & Eko & $6-15$ \\
\hline 3 & Orthic Humiluvic Spodosol & Eko & $45-81$ \\
\hline 4 & Orthic Humiluvic Spodosol & Eko & $81-103$ \\
\hline 5 & Orthic Humiluvic Spodosol & Eko & $107-132$ \\
\hline 6 & Orthic Natric Planosol & SNo & $0-2$ \\
\hline 7 & Orthic Natric Planosol & SNo & $2-8$ \\
\hline 8 & Orthic Natric Planosol & SNo & $25-36$ \\
\hline 9 & Orthic Natric Planosol & SNo & $47-75$ \\
\hline 10 & Orthic Quartzarenic Neosol & RQo & $0-12$ \\
\hline 11 & Orthic Quartzarenic Neosol & RQo & $36-58$ \\
\hline 12 & Orthic Quartzarenic Neosol & RQo & $58-86$ \\
\hline 13 & Orthic Quartzarenic Neosol & RQo & $131-145$ \\
\hline 14 & Orthic Quartzarenic Neosol & RQo & $0-12$ \\
\hline 15 & Orthic Quartzarenic Neosol & RQo & $20-40$ \\
\hline 16 & Orthic Quartzarenic Neosol & RQo & $40-80$ \\
\hline 17 & Orthic Quartzarenic Neosol & RQo & $80-125$ \\
\hline 18 & Orthic Quartzarenic Neosol & RQo & $0-4$ \\
\hline 19 & Orthic Quartzarenic Neosol & RQo & $4-30$ \\
\hline 20 & Orthic Quartzarenic Neosol & RQo & $30-60$ \\
\hline
\end{tabular}

Table 1. Soil collection selected for this study and classified according to the Brazilian System of Soil Classification (Embrapa, 2013). 
Orthic Quartzarenic Neosol Orthic Quartzarenic Neosol Orthic Quartzarenic Neosol Orthic Quartzarenic Neosol Orthic Quartzarenic Neosol Orthic Quartzarenic Neosol Orthic Haplic Chernosol Orthic Haplic Chernosol Orthic Haplic Chernosol

Orthic Haplic Chernosol Eutrophic Regolitic Neosol Eutrophic Regolitic Neosol Eutrophic Regolitic Neosol Eutrophic Regolitic Neosol Eutrophic Haplic Gleisol Eutrophic Haplic Gleisol Eutrophic Haplic Gleisol Eutrophic Haplic Gleisol Orthic Haplic Vertisol Orthic Haplic Vertisol Orthic Haplic Vertisol Orthic Haplic Vertisol Saprolitic Carbonatic Haplic Cambisol Saprolitic Carbonatic Haplic Cambisol Saprolitic Carbonatic Haplic Cambisol

Orthic Quartzarenic Neosol Orthic Quartzarenic Neosol Orthic Quartzarenic Neosol Orthic Quartzarenic Neosol Eutrophic Haplic Planosol Eutrophic Haplic Planosol Eutrophic Haplic Planosol Petrocalcic Rendzic Chernosol Petrocalcic Rendzic Chernosol Petrocalcic Rendzic Chernosol Petrocalcic Rendzic Chernosol Tipic Eutrophic Red Nitosol Tipic Eutrophic Red Nitosol Tipic Eutrophic Red Nitosol Tipic Eutrophic Red Nitosol Dystroferric Red Latosol Dystroferric Red Latosol Dystroferric Red Latosol Dystroferric Red Latosol Quartzarenic Neosol Quartzarenic Neosol Quartzarenic Neosol Quartzarenic Neosol Quartzarenic Neosol
RQo

RQo

RQo

RQo

MXo

MXo

MXo

MXo

RRe

$\mathrm{RRe}$

$\mathrm{RRe}$

$\mathrm{RRe}$

GXve

GXve

GXve

GXve

VXo

VXo

VXo

VXo

CXk

$\mathrm{CXk}$

CXk

RQo

RQo

RQo

RQo

SXe

SXe

SXe

MDlk

MDlk

MDlk

MDlk

Nve

Nve

Nve

Nve

LVdf

LVdf

LVdf

LVdf

RQ

RQ

RQ

RQ

RQ
60-103

56-78

88-107

107-128

$0-10$

10-29

29-50

50-72

0-10

28-63

91-125

$0-4$

18-45

64-91

$0-4$

16-26

34-57

$0-5$

30-55

0-5

9-19

$19-53$

53-80

$0-4$

$30-45$

45-87

0-8

8-24

$24-41$

41-61

61-72

10-23

23-39

$39-59$

0-7

07-14

$29-47$

47-70

$0-12$

12-27

$27-47$

47-73

73-118

Table 2. Soil attributes selected to estimate the field capacity (FC).

\begin{tabular}{|c|c|c|c|c|c|}
\hline $\mathrm{N}^{\mathrm{o}}$ & Clay & Sand & Organic matter & Coarse sand & Microporosity \\
\hline \multicolumn{6}{|c|}{${ }-{ }$} \\
\hline 2 & 4 & 90 & 0.8 & 23 & 37 \\
\hline 4 & 6 & 91 & 0.4 & 23 & 21 \\
\hline 5 & 14 & 82 & 0.1 & 18 & 25 \\
\hline 6 & 6 & 58 & 10.1 & 19 & 50 \\
\hline
\end{tabular}




\begin{tabular}{|c|c|c|c|c|c|}
\hline 7 & 10 & 60 & 2.5 & 20 & 48 \\
\hline 8 & 6 & 72 & 0.1 & 21 & 28 \\
\hline 9 & 35 & 38 & 0.2 & 10 & 38 \\
\hline 10 & 4 & 93 & 1.0 & 29 & 21 \\
\hline 11 & 4 & 92 & 0.2 & 28 & 20 \\
\hline 12 & 4 & 95 & 0.1 & 30 & 20 \\
\hline 13 & 4 & 95 & 0.0 & 30 & 24 \\
\hline 14 & 4 & 93 & 0.8 & 30 & 24 \\
\hline 15 & 4 & 94 & 0.2 & 29 & 22 \\
\hline 16 & 4 & 94 & 0.2 & 29 & 22 \\
\hline 17 & 4 & 95 & 0.1 & 29 & 23 \\
\hline 18 & 6 & 63 & 3.8 & 12 & 30 \\
\hline 19 & 8 & 82 & 1.0 & 16 & 27 \\
\hline 20 & 10 & 84 & 0.5 & 17 & 27 \\
\hline 21 & 8 & 86 & 0.3 & 20 & 32 \\
\hline 22 & 4 & 91 & 1.2 & 13 & 35 \\
\hline 23 & 4 & 93 & 0.5 & 13 & 32 \\
\hline 24 & 4 & 95 & 0.0 & 14 & 26 \\
\hline 25 & 6 & 88 & 0.1 & 12 & 28 \\
\hline 26 & 6 & 88 & 0.0 & 11 & 28 \\
\hline 27 & 25 & 57 & 2.2 & 37 & 33 \\
\hline 28 & 27 & 56 & 1.8 & 37 & 32 \\
\hline 29 & 25 & 58 & 1.0 & 38 & 29 \\
\hline 30 & 25 & 54 & 0.5 & 35 & 29 \\
\hline 31 & 10 & 73 & 2.1 & 63 & 20 \\
\hline 32 & 10 & 76 & 0.8 & 64 & 15 \\
\hline 33 & 10 & 69 & 0.5 & 61 & 14 \\
\hline 34 & 10 & 80 & 0.2 & 63 & 17 \\
\hline 35 & 38 & 23 & 5.7 & 14 & 35 \\
\hline 36 & 36 & 34 & 2.8 & 21 & 28 \\
\hline 37 & 21 & 50 & 0.5 & 32 & 33 \\
\hline 38 & 23 & 45 & 0.2 & 30 & 29 \\
\hline 39 & 32 & 32 & 5.9 & 22 & 31 \\
\hline 40 & 34 & 39 & 3.5 & 21 & 35 \\
\hline 41 & 40 & 32 & 1.9 & 20 & 31 \\
\hline 42 & 44 & 33 & 0.8 & 21 & 37 \\
\hline 43 & 47 & 11 & 8.8 & 7 & 40 \\
\hline 44 & 55 & 11 & 4.1 & 6 & 38 \\
\hline 45 & 64 & 12 & 1.2 & 6 & 38 \\
\hline 46 & 4 & 84 & 1.3 & 13 & 32 \\
\hline 47 & 4 & 91 & 0.3 & 14 & 23 \\
\hline 48 & 4 & 93 & 0.1 & 14 & 21 \\
\hline 49 & 4 & 92 & 0.1 & 16 & 22 \\
\hline 50 & 12 & 43 & 4.1 & 4 & 31 \\
\hline 51 & 38 & 39 & 0.4 & 6 & 29 \\
\hline 52 & 36 & 44 & 0.3 & 8 & 29 \\
\hline 53 & 17 & 54 & 8.5 & 19 & 51 \\
\hline 54 & 21 & 53 & 6.9 & 15 & 45 \\
\hline 55 & 19 & 58 & 5.2 & 19 & 43 \\
\hline 56 & 11 & 73 & 4.4 & 33 & 45 \\
\hline 57 & 58 & 17 & 3.4 & 8 & 31 \\
\hline 58 & 60 & 16 & 2.6 & 9 & 35 \\
\hline 59 & 66 & 14 & 1.9 & 7 & 39 \\
\hline 60 & 74 & 12 & 1.5 & 6 & 41 \\
\hline 61 & 61 & 16 & 4.3 & 7 & 31 \\
\hline 62 & 63 & 16 & 3.6 & 8 & 35 \\
\hline 63 & 66 & 19 & 2.2 & 8 & 40 \\
\hline 64 & 66 & 20 & 1.8 & 8 & 42 \\
\hline 65 & 10 & 88 & 1.0 & 41 & 18 \\
\hline 66 & 10 & 89 & 0.4 & 40 & 15 \\
\hline
\end{tabular}


Assessing pedotransfer...

RIBEIRO, B. T. et al.

\begin{tabular}{|c|c|c|c|c|c|}
\hline 67 & 12 & 86 & 0.3 & 34 & 13 \\
\hline 68 & 12 & 85 & 0.3 & 32 & 14 \\
\hline 69 & 14 & 85 & 0.2 & 32 & 13 \\
\hline Average & 22 & 63 & 1.8 & 22 & 30 \\
\hline $\begin{array}{l}\text { Standard } \\
\text { deviation }\end{array}$ & 21 & 30 & 2.3 & 14 & 9 \\
\hline
\end{tabular}

$* \%$ unit was used to respect the original equations (1,2 and 3). According to International System of Units (SI), for total sand, clay, coarse sand and organic matter $1 \%=10 \mathrm{~g} \mathrm{~kg}^{-1}$ and for microporosity $1 \%=\left(\mathrm{cm}^{3} \mathrm{~cm}^{-3}\right) \cdot 100$

\section{RESULTS AND DISCUSSION}

PCA identified four soil groups (Figure 1). The soil groups were classified by texture. The first group included soils with a clay content ranging from $6.2 \%$ to $35.2 \%$ : surface and subsurface samples from Planosols and Chernosols and subsurface samples from Gleisols. The second group included clay soils (clay content ranging from $35.2 \%$ to $74.1 \%$ ): surface and subsurface samples from Gleisols, Vertisols, Cambisols, Nitosols, and Latosols, and subsurface samples from Planosols.
The third and fourth groups included sandy soils (clay content of less than $15.0 \%$ ). The third group was formed by surface and subsurface samples from Spodosols and Orthic Quartzarenic Neosols, and by subsurface samples (depth of 25 to $36 \mathrm{~cm}$ ) from Orthic Natric Planosols. The fourth group comprised soils with the highest coarse sand content: surface and subsurface samples from Orthic Quartzarenic Neosols, Quartzarenic Neosols, Eutrophic Regolithic Neosol and one subsurface sample (depth of 45 to $81 \mathrm{~cm}$ ) from Orthic Humiluvic Spodosol.

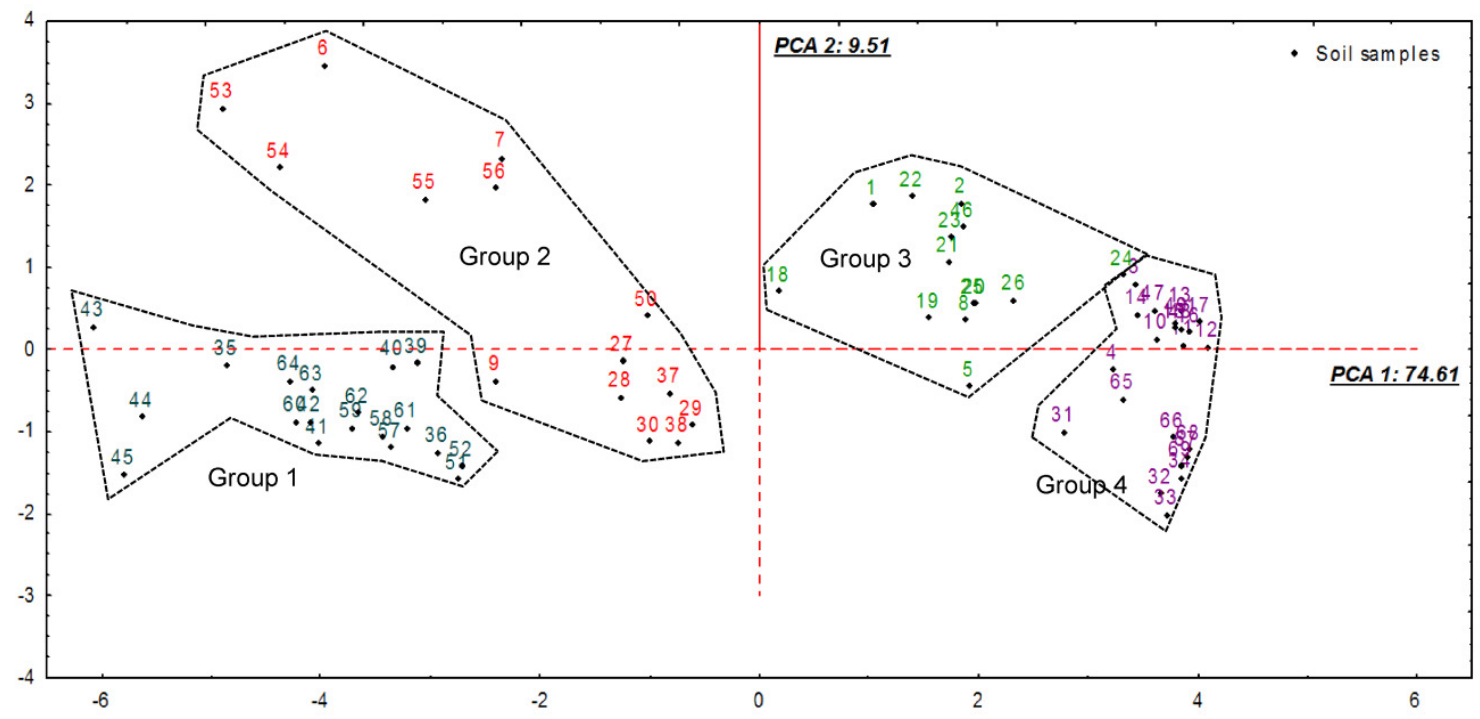

Figure 1. Principal component analysis (PCA 1 and PCA 2) of the studied soils. The dots and numbers represent the soil order. Group 1: 6, 7, 9, 27, 28, 29, 30, 37, 38, 50, 53, 54, 55, and 56. Group 2: 9, $35,36,39,40,41,42,43,44,45,51,52,57,58,59,60,61,62,63$, and 64. Group 3: 1, 2, 4, 5, 8, 19 , $20,21,22,23,25,26$, and 46. Group 4: 3, 10, 11, 12, 13, 14, 15, 16, 17, 24, 31, 32, 33, 34, 47, 48, $49,65,66,67,68$, and 69 .

PCA indicated that FC estimated by PF-1 was closer to soil moisture at $10 \mathrm{KPa}$ and $33 \mathrm{KPa}$ compared with FC estimated by PF-2 and PF-3 (Figure 2). In addition, the results of the MannWhitney rank-sum test revealed that there was no statistically significant difference between the FC estimated by PTFs 1, 2, and 3, and the soil moisture remaining at $10 \mathrm{KPa}$ and $33 \mathrm{KPa}$ (except for PTF-1 at $33 \mathrm{KPa}$ ). Moreover, there was a negative correlation between sand content (total and coarse) and soil water retention. The clay content was more related to the remaining soil moisture at higher potentials. 


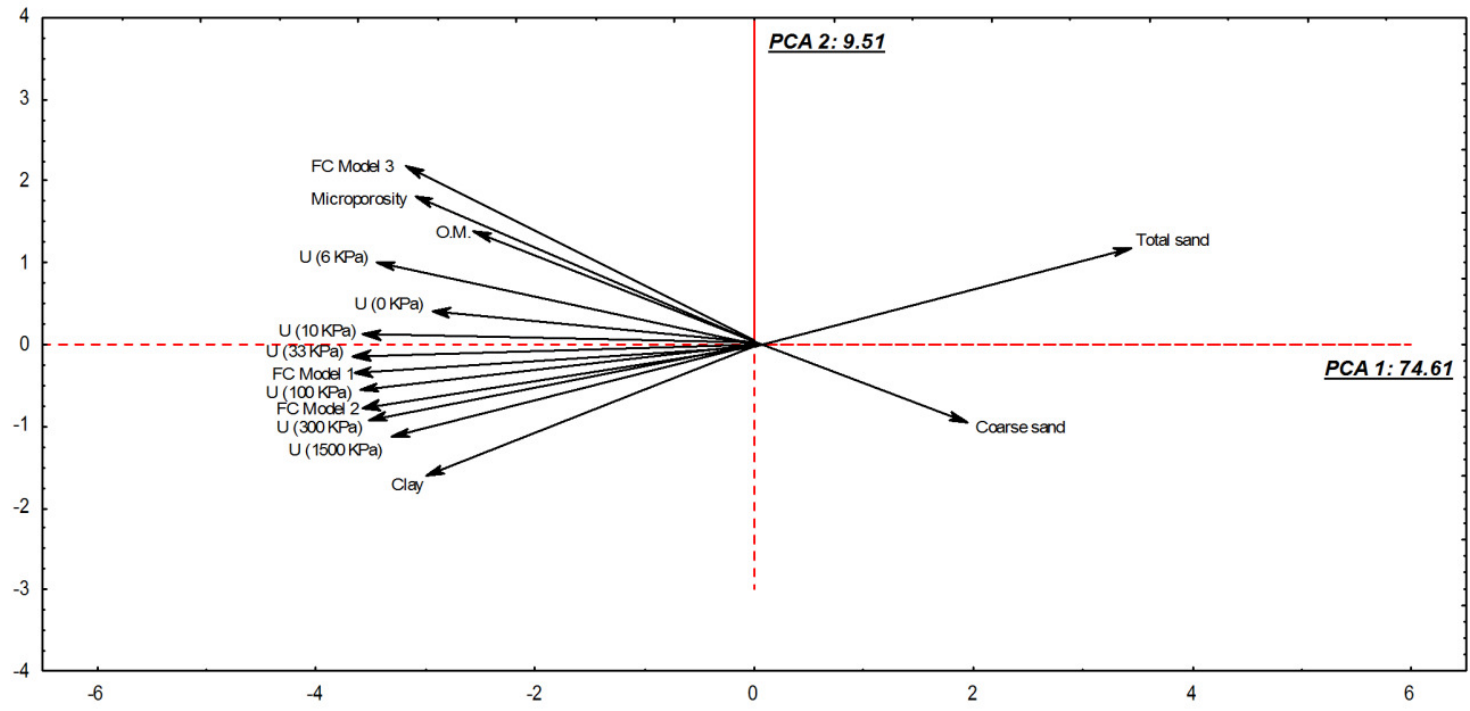

Figure 2. Principal component analysis (PCA 1 and PCA 2) of the soil attributes and FC estimated by PTFs 1 , 2 , and 3 (the order is represented by the arrows).

Table 2. Mann-Whitney rank-sum test (p-value) comparing field capacity (FC) estimated by PTFs 1, 2, and 3 (MACEDO, 1991) and the soil moisture remaining at different potentials.

\begin{tabular}{cccccccc}
\hline \multirow{2}{*}{ PF } & \multicolumn{7}{c}{ Applied tension $(\mathrm{KPa})$} \\
\cline { 2 - 7 } & 0 & 6 & 10 & 33 & 100 & 300 & 1.500 \\
\cline { 2 - 7 } & $<0.001^{*}$ & $0.003^{*}$ & $0.950^{\mathrm{ns}}$ & $0.026^{*}$ & $<0.001^{*}$ & $<0.001^{*}$ & $<0.001^{*}$ \\
2 & $<0.001^{*}$ & $0.003^{*}$ & $0.392^{\mathrm{ns}}$ & $0.204^{\mathrm{ns}}$ & $<0.001^{*}$ & $<0.001^{*}$ & $<0.001^{*}$ \\
3 & $<0.001^{*}$ & $<0.001^{*}$ & $0.714^{\mathrm{ns}}$ & $0.134^{\mathrm{ns}}$ & $0.002^{*}$ & $<0.001^{*}$ & $<0.001^{*}$ \\
\hline
\end{tabular}

*statistically significant difference: the difference in the median values between the two groups is higher than would be expected by chance; ns, no statistically significant difference: the difference in the median values between the two groups may be due to random sampling variability.

FC values estimated by PTFs 1, 2, and 3 and correlated with the soil water contents measured at different potentials $(\mathrm{kPa})$ are shown in Figures 3, 4, and 5, respectively. The best fit (1:1 regression line) for all PTFs was obtained for estimated FC values and soil moisture at $10 \mathrm{kPa}$. A potential of $10 \mathrm{kPa}$ or $33 \mathrm{kPa}$ is commonly used to determine the water holding capacity of soils (upper limit of the water content available to plants). Nonetheless, there is not a consensus about the optimal potential to be applied to different soils. For instance, soil water retention at $10 \mathrm{kPa}$ or $33 \mathrm{kPa}$ did not represent the upper water limit available under field conditions for cohesive horizons from Yellow Latosol (AGUIAR NETTO et al., 1999).

The PTFs proposed by Macedo (1991) were obtained for sandy surface horizons from Argisols. In this study, the mean and median concentration of sand in the soil collection used was $63 \%$ and $72 \%$, respectively (Table 2). These results may help explain the suitability of all tested PTFs to estimate FC (Figures 3, 4, and 5). The tested PFs were also appropriately correlated with the determined FC in situ (FABIAN; OTTONI FILHO, 2000).
The predicted FC values allowed estimating the soil water content remaining at the applied potentials. All PTFs were significant (F test) with $\mathrm{R}^{2}$ ranging from 0.42 to 0.83 , indicating that the water content remaining at different tensions (from 0 to $1500 \mathrm{KPa}$ ) was associated with $\mathrm{FC}$ values estimated using the three PTFs. Ghanbarian-Alavijeh and Millán (2010) also found that the water contents at different matric potentials were linearly correlated with each other. 

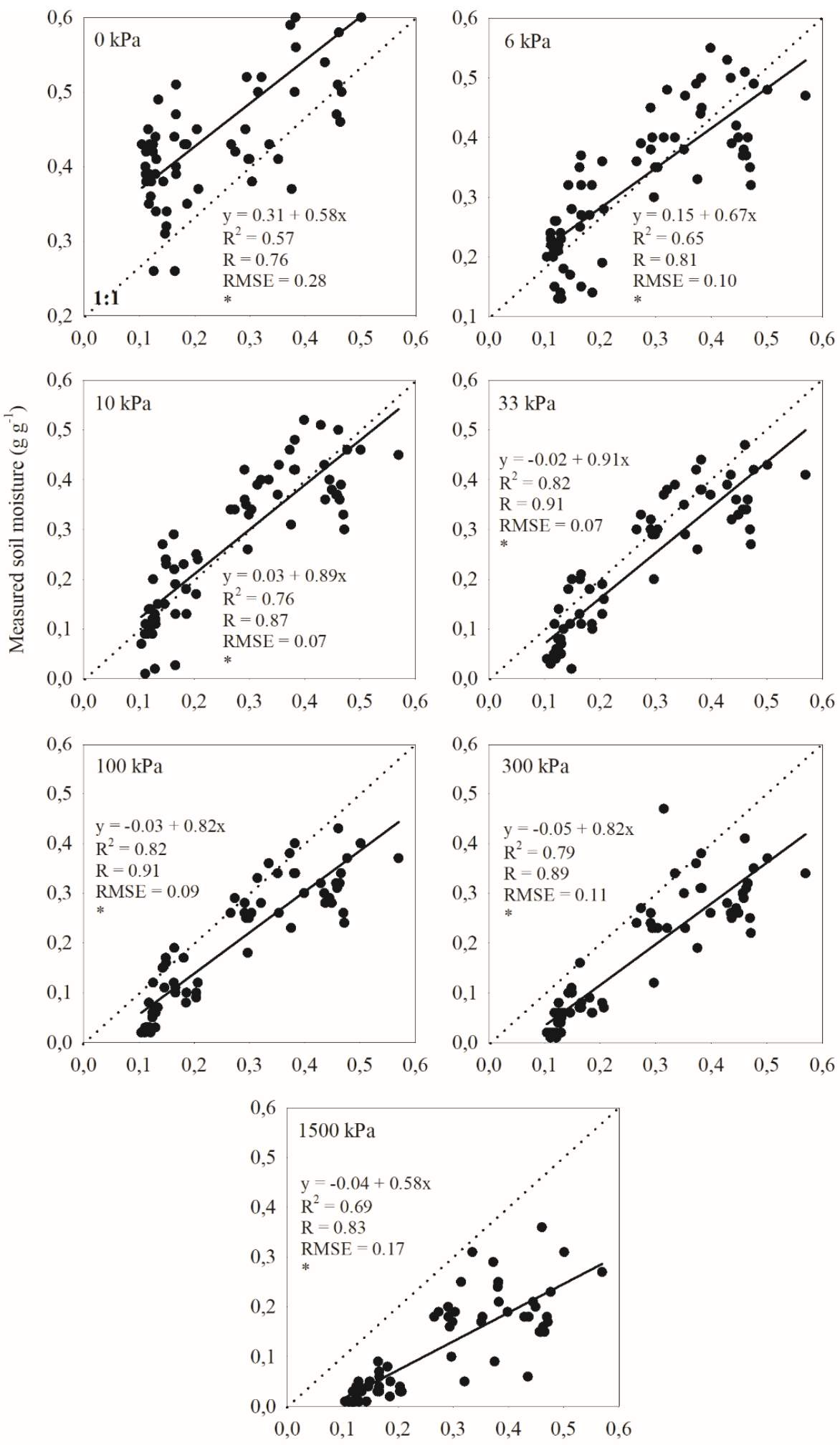

Estimated field capacity $\left(\mathrm{cm}^{3} \mathrm{~cm}^{-3}\right)$

Figure 3. Linear regression between field capacity (FC) estimated by PTF-1 and soil moisture measured at different tensions $(0,6,10,33,100,300$, and $1500 \mathrm{KPa})$. The dotted line represents the $1: 1$ regression line. ${ }^{*} \mathrm{p}<0.001$ ( $\mathrm{F}$ test). 

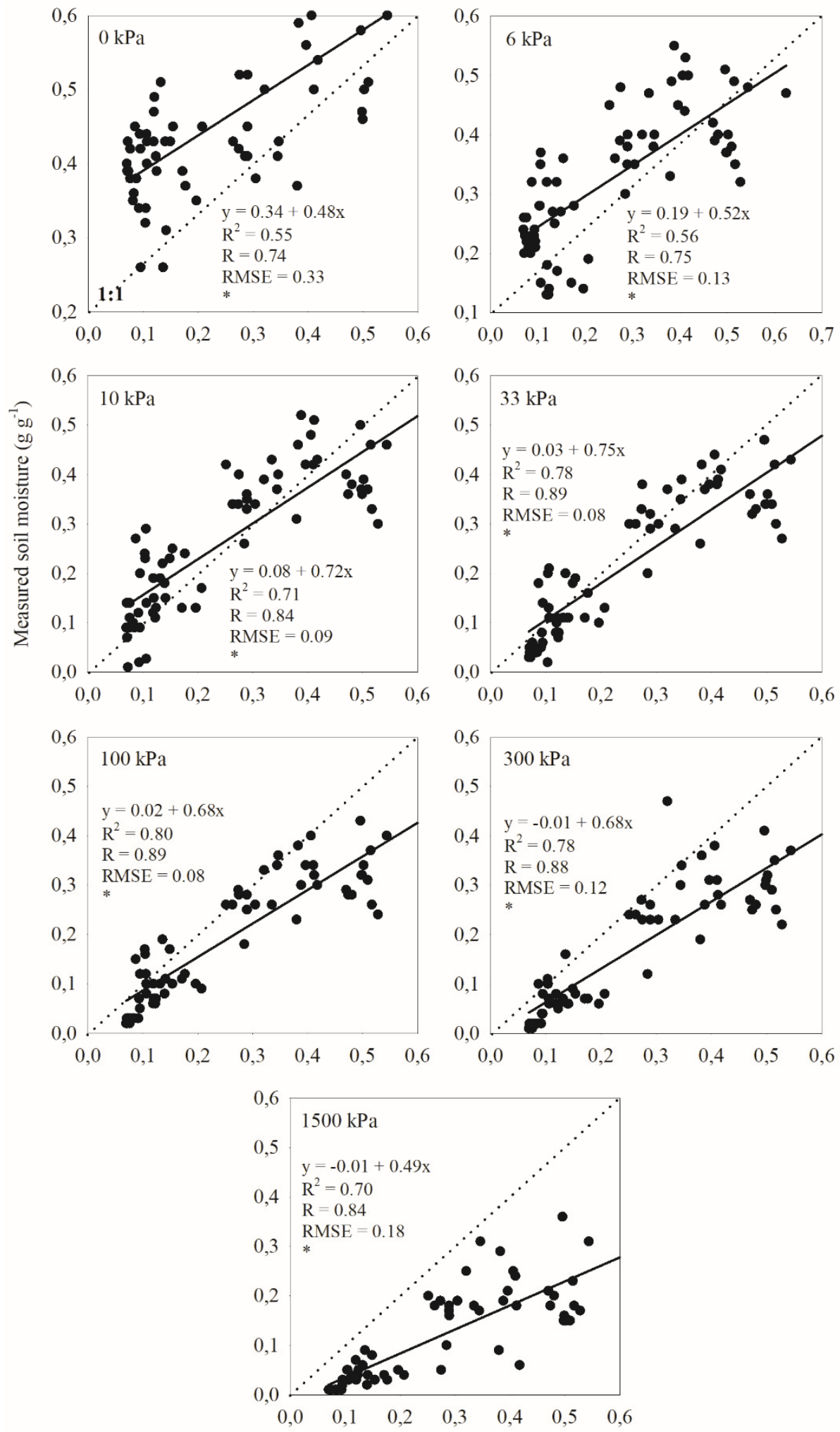

Estimated field capacity $\left(\mathrm{cm}^{3} \mathrm{~cm}^{-3}\right)$

Figure 4. Linear regression between field capacity (FC) estimated using PTF-2 and soil moisture quantified at different tensions: $0,6,10,33,100,300$, and $1500(\mathrm{KPa})$. The dotted line represents the 1:1 regression line. ${ }^{*} \mathrm{p}<0.001$ (F test). 

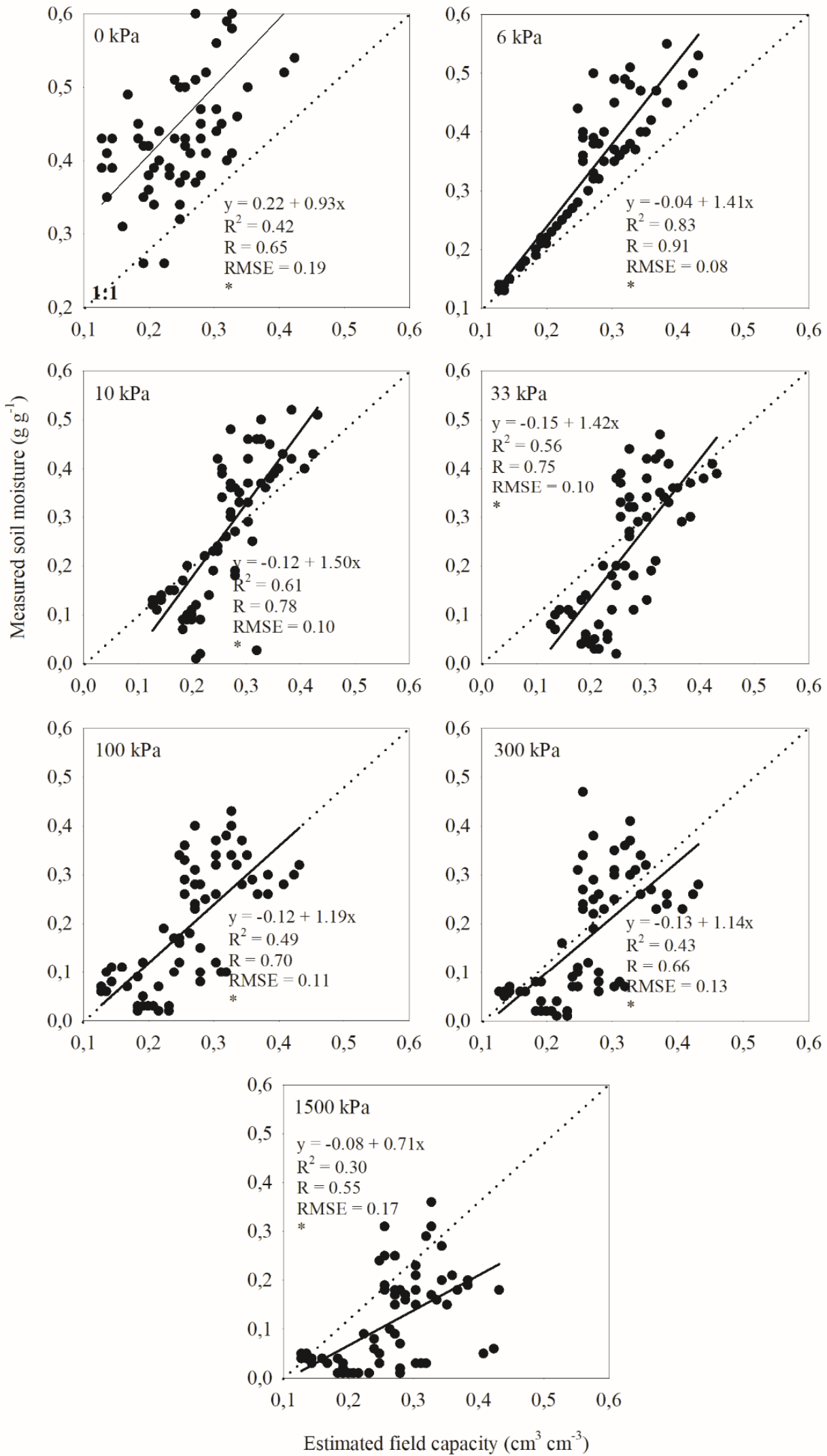

Figure 5. Linear regression between field capacity (FC) estimated using PF3 and soil moisture measured at different tensions $(\mathrm{KPa}): 0,6,10,33,100,300$, and 1500. The dotted line represents the 1:1 regression line. ${ }^{*} \mathrm{p}<0.001$ (F test). 
After four soil groups were identified by PCA, PTFs 1, 2, and 3 were reapplied in each soil group to estimate $\mathrm{FC}$ at $10 \mathrm{KPa}$ and $33 \mathrm{KPa}$ (Table $3)$. In group 1 , the three PTFs accurately predicted $\mathrm{FC}$ at $33 \mathrm{KPa}$. In group 2, PTF-1 accurately predicted $\mathrm{FC}$ at $10 \mathrm{KPa}$. In group 3, PTF-1 accurately estimated $\mathrm{FC}$ at $33 \mathrm{KPa}$. In group 4, PTFs 1 and 2 appropriately estimated FC at $10 \mathrm{KPa}$. PTFs 1 and 2 presented a larger variation than PTF3 (equations 1 to 3 ). PTF-3 predicted only microporosity and was suitable for only one condition (group 1 soils at $10 \mathrm{KPa}$ ).

Table 3. Mann-Whitney rank-sum test (p-value) comparing field capacity (FC) estimated by PTFs 1,2 , and 3 (Macedo, 1991) and the soil moisture content remaining at $10 \mathrm{KPa}$ and $33 \mathrm{KPa}$ for each soil group identified by PCA.

\begin{tabular}{|c|c|c|c|c|c|c|c|c|}
\hline \multirow[b]{2}{*}{$\mathrm{PF}$} & \multicolumn{8}{|c|}{ Tension $(\mathrm{kPa})$} \\
\hline & 10 & 33 & 10 & 33 & 10 & 33 & 10 & 33 \\
\hline & \multicolumn{2}{|c|}{ Group 1} & \multicolumn{2}{|c|}{ Group 2} & \multicolumn{2}{|c|}{ Group 3} & \multicolumn{2}{|c|}{ Group 4} \\
\hline 1 & $0.03 *$ & $0.77^{\mathrm{ns}}$ & $0.133^{\mathrm{ns}}$ & $0.002 *$ & $0.002 *$ & $0.47^{\mathrm{ns}}$ & $0.15^{\mathrm{ns}}$ & $<0.001 *$ \\
\hline 2 & $0.009 *$ & $0.48^{\mathrm{ns}}$ & $0.007 *$ & $<0.001 *$ & $<0.001 *$ & $0.04 *$ & $0.83^{\mathrm{ns}}$ & $0.002 *$ \\
\hline 3 & $0.05^{*}$ & $0.63^{\text {ns }}$ & $<0.001 *$ & $<0.001 *$ & $0.02 *$ & $<0.001 *$ & $<0.001 *$ & $<0.001 *$ \\
\hline
\end{tabular}

* statistically significant difference: the difference in the median values between the two groups was higher than would be expected by chance; ns, no statistically significant difference: the difference in the median values between the two groups may be due to random sampling variability.

\section{CONCLUSIONS}

Considering the full dataset, a good 1:1 ratio between estimated field capacity and observed soil water retention at $10 \mathrm{kPa}$ was obtained.

Predicted field capacity was statistically similar to measured values. Principal component analysis identified four different soil groups based on texture.
After reapplying the pedotransfer functions to each soil group, the difference between predicted and measured values increased.

\section{ACKNOWLEDGEMENTS}

The authors gratefully acknowledge the following Brazilian research agencies: Fapemig, Capes and CNPq. Also, we thank CNPq (Process 141595/2018-3) supporting the costs of this publication.

RESUMO: Considerando a importância da retenção de água no solo para fins agronômicos e ambientais, objetivou-se com este trabalho avaliar três funções de pedotransferência (FP) para estimativa da capacidade de campo (CC) com base em atributos de solo facilmente determinados. Uma coleção de 17 solos dos biomas Cerrado e Pantanal, incluindo amostras superficiais e subsuperficiais, foram utilizadas. FP 1 considera o conteúdo de argila, matéria orgânica, areia grossa e microporosidade. FP 2 considera argila, areia total e matéria orgânica. A FP 3 leva em consideração apenas microporosidade. Os valores estimados de $\mathrm{CC}$ foram correlacionados aos valores de umidade obtidos em diferentes potenciais $(0,6,10,33,100,300$, and $1500 \mathrm{kPa})$ com o intuito de verificar qual potencial corresponde à CC estimada. Os dados foram submetidos à análise de regressão, ao teste Mann-Whitney rank-sum para comparar valores medidos e estimados e realizada análise de componentes principais (PCA). Considerando todo o conjunto de dados, foi obtida uma forte correlação ( $\mathrm{R}$ 0.84-0.91; $\mathrm{R}^{2}$ 0.71-0.82; RMSE 0.07-0.09) entre CC estimada e a umidade do solo obtida nos potenciais de $10 \mathrm{kPa}$ e $33 \mathrm{kPa}$. A CC estimada pela FP 3 correlacionou melhor com a retenção de água no potencial de 6 $\mathrm{kPa}$. Quando as FP's foram reaplicadas em grupos de solos homogêneos (identificados pela PCA), a correlação entre valores estimados e medidos diminuiu.

PALAVRAS-CHAVE: Retenção de água no solo. Análise de componentes principais. Umidade do solo.

\section{REFERENCES}

AGUIAR NETTO, A. O.; NACIF, P. G. S.; REZENDE, J. O. Avaliação do conceito de capacidade de campo para um Latossolo Amarelo coeso do Estado da Bahia. Revista Brasileira de Ciência do solo, v. 23, n. 3, p. 661-667, 1999. https://doi.org/10.1590/S0100-06831999000300020 
BOTULA, Y. D.; RANST, E. V.; CORNELIS, W. M. Pedotransfer functions to predict water retention for soils of the humid tropics: A review. Revista Brasileira de Ciência do Solo, v. 38, n. 3, p. 679-698, 2014. https://doi.org/10.1590/S0100-06832014000300001

BOUMA, J. Using soil survey data for quantitative land evaluation. In: STEWART, B.A. (Ed.) Advances in Soil Science. New York: Springer Verlag. 1989. v. 9. p. 177-213. https://doi.org/10.1007/978-1-4612-3532-3_4

COSTA, A.; ALBUQUERQUE, J. A.; ALMEIDA, J. A.; COSTA, A.; LUCIANO, R. V. Pedotransfer functions to estimate retention and availability of water in soils of the State of Santa Catarina, Brazil. Revista Brasileira de Ciência do Solo, v. 37, p. 889-910, 2013. https://doi.org/10.1590/S0100-06832013000600010 https://doi.org/10.1590/S0100-06832013000400007

FABIAN, A. J.; OTTONI FILHO, T. B. Determinação de capacidade de campo in situ ou através de equações de regressão. Pesquisa Agropecuária Brasileira, v. 35, n. 5, p. 1029-1036, 2000.

https://doi.org/10.1590/S0100-204X2000000500022

GHANBARIAN-ALAVIJEH, B.; LIAGHAT, A.; HUANG, G. H.; VAN GENUCHTEN, M.TH. Estimation of the van Genuchten soil water retention properties from soil textural data. Pedosphere, v. 20, n. 4, p. 456-465, 2010. https://doi.org/10.1016/S1002-0160(10)60035-5

HARTEMINK, A. E. Soil science in tropical and temperate regions - some differences and similarities. Advances in Agronomy, v. 77, p. 269-292, 2002. https://doi.org/10.1016/S0065-2113(02)77016-8

KLEIN, V. A.; REICHERT, J. M.; REINERT, D. J. Água disponível em um Latossolo Vermelho argiloso e murcha fisiológica de culturas. Revista Brasileira de Engenharia Agrícola e Ambiental, v. 10, p. 646-650, 2006. https://doi.org/10.1590/S1415-43662006000300016

MACEDO, J. R. Determinação de retenção hídrica por correlação múltipla e de variabilidade espacial em solos podzólicos de Seropédica, RJ. 1991. 174 f. Dissertação, Universidade Federal Rural do Rio de Janeiro, Rio de Janeiro, 1991. https://doi.org/10.1081/CSS-120005747

MACEDO, J. R.; MENEGUELLI, N. A.; OTTONI FILHO, T. B.; LIMA, J. R. S. Estimation of field capacity and moisture retention based on regression analysis involving chemical and physical properties in Alfisols and Ultisols of the state of Rio de Janeiro. Communications in Soil Science and Plant Analysis, v. 33, p. 2037-2055, 2002.

MINASNY, B.; HARTEMINK, A. E. Predicting soil properties in the tropics. Earth-Science Reviews, v. 106, p. 52-62, 2011. https://doi.org/10.1016/j.earscirev.2011.01.005

OKI, T.; KANAE, S. Global hydrological cycles and world water resources. Science, v. 313, n. 5790, p. 1068 1072, 2006. https://doi.org/10.1126/science.1128845

PIDGEON, J. D. The measurement and prediction of available water capacity of ferrallitic soils in Uganda. Journal of Soil Science, v. 23, n. 4, p. 431- 441, 1972. https://doi.org/10.1111/j.1365-2389.1972.tb01674.x

RAWLS, W. J.; PACHEPSKY, Y. A.; RITCHIE, J. C.; SOBECKI, T. M.; BLOODWORTH, H. Effect of soil organic carbon on soil water retention. Geoderma, v. 116, p. 61-76, 2003. https://doi.org/10.1016/S00167061(03)00094-6

REICHARDT, K. Capacidade de campo. Revista Brasileira de Ciência do Solo, v. 12, p. 211-216, 1988.

REICHERT, J. M.; ALBUQUERQUE, J. A.; KAISER, D. R.; REINERT, D. J.; URACH, F. L.; CARLESSO, R. Estimation of water retention and availability in soils of Rio Grande do Sul. Revista Brasileira de Ciência do Solo, v. 33, n. 6, p. 1547-1560, 2009. https://doi.org/10.1590/S0100-06832009000600004 
REUNIÃO BRASILEIRA DE CLASSIFICAÇÃO E CORRELAÇÃO DE SOLOS, 10., 2012. Corumbá. Guia de excursão de estudos de solos no Pantanal e Cerrados do estado de Mato Grosso do Sul. Corumbá: Embrapa Pantanal; Rio de Janeiro: Embrapa Solos; Campo Grande: Embrapa Gado de Corte; Viçosa: Sociedade Brasileira de Ciência do Solo; Recife: Ed. da Universidade Federal Rural de Pernambuco, 2012. $176 \mathrm{p}$.

RUIZ, H. A.; FERREIRA, G. B.; PEREIRA, J. B. M. Estimativa da capacidade de campo de Latossolos e Neossolos Quartzarênicos pela determinação do equivalente de umidade. Revista Brasileira de Ciência do Solo, v. 27, p. 389-393, 2003. https://doi.org/10.1590/S0100-06832003000200019

SANTOS, W. J. R.; CURI, N.; SILVA, S. H. G.; ARAÚJO, E. F.; MARQUES, J. J. Pedotransfer functions for water retention in different soil classes from the center- southern Rio Grande do Sul State. Ciência e Agrotecnologia, v. 37, n. 1, p. 49-60, 2013. https://doi.org/10.1590/S1413-70542013000100006

SILVA, B. M.; SILVA, E.É. A.; OLIVEIRA, G. C.; FERREIRA, M. M.; SERAFIM, M. E. Plant-available soil water capacity: Estimation methods and implications. Revista Brasileira de Ciência do Solo, v. 38, n. 1, p. 464-475, 2014. https://doi.org/10.1590/S0100-06832014000200011

SOUZA, L. D.; REICHARDT, K. Estimativas da capacidade de campo. Revista Brasileira de Ciência do Solo, v. 20, n. 2, p. 183-189, 1996.

TOLK, J. A. Plant-available Soil Water In: LAL, R. (Ed.). Encyclopedia of Water Science. New York: Marcel Dekker, Ic., 2008. p. 1113-1116.

TOMASELLA, J.; HODNETT, M. G.; ROSSATO, L. Pedotransfer functions for the estimation of soil water retention in Brazilian soils. Soil Science Society of American. Journal, v. 64, p. 327-338, 2000. https://doi.org/10.2136/sssaj2000.641327x

VEIHMEYER, F. J.; HENDRICKSON, A. H. Methods of measuring field capacity and permanent wilting percentages of soils. Soil Science, v. 68, n. 1, p. 75-94, 1949. https://doi.org/10.1097/00010694-19490700000007 